\title{
Growth kinetics and metronidazole sensitivity of Blastocystis spp. isolated from colorectal carcinoma (CRC) and non-CRC patients
}

\author{
Fayza SM Habib, Nashwa S Abdel-Fattah, Ghada A Saad, Heba M El Naggar \\ Medical Parasitology Department, Faculty of Medicine, Ain-Shams University, Cairo, Egypt
}

\begin{abstract}
Background: Blastocystis spp. is the most common protist detected in human fecal samples in numerous studies globally. Until recently debate regarding its pathogenicity has been controversial. Some studies speculated a possible correlation between Blastocystis and colorectal carcinoma (CRC). No previous studies investigated the presence of non-genotypic differences in Blastocystis spp. isolated from CRC and non-CRC patients.

Objective: The present work aimed to investigate the growth kinetics (GK) and metronidazole (MTZ) sensitivity of Blastocystis isolates from CRC and apparent non-CRC symptomatic and asymptomatic Blastocystis carriers.

Material and Methods: Seven isolates from CRC patients, 6 from symptomatic and 6 from asymptomatic non-CRC carriers were cultured in Locke's Egg (LE) medium supplemented with bovine serum and antibiotic mixture, and incubated at $37^{\circ} \mathrm{C}$. Mean viable organisms counts of each isolate were calculated every $24 \mathrm{~h}$ to follow their GK. Counts were repeated after exposure to different MTZ concentrations to test their drug sensitivity.

Results: In vitro GK of CRC and non-CRC symptomatic isolates were nearly similar at $72 \mathrm{~h}$ incubation with apparently higher peaks attained by isolates from CRC patients than the slower growing non-CRC asymptomatic isolates. MTZ-sensitivity of CRC isolates was nearly similar to that of non-CRC asymptomatic isolates; both were significantly more sensitive than the symptomatic isolates especially at high drug concentration of $200 \mu \mathrm{g} / \mathrm{ml}$ of the medium.

Conclusion: The recorded difference in GK and MTZ sensitivity of Blastocystis spp. isolated from different clinical groups suggests the existence of certain biological characters with different pathogenic roles.
\end{abstract}

Keywords: Asymptomatic, Blastocystis spp., colorectal carcinoma, growth kinetics, metronidazole sensitivity, symptomatic.

Received: 18 November, 2018, Accepted: 12 December, 2018.

Corresponding Author: Ghada A Saad, Tel.: 01005818392, E-mail: dr_ghadasaad@yahoo.com

Print ISSN: 1687-7942, Online ISSN: 2090-2646, Vol. 11, No. 3, December, 2018.

\section{INTRODUCTION}

Blastocystis spp. are the most common protists detected in human fecal samples in numerous studies globally $^{(1,2)}$. It is a pleomorphic organism existing in multiple forms: vacuolar, granular, amoeboid and cystic $^{(3)}$. The debate regarding Blastocystis spp. pathogenicity led many researchers to attempt identification of differentiating characters between asymptomatic and symptomatic human-derived isolates $^{(4)}$. Different growth profiles characterizing isolates from different clinical groups were previously reported $^{(4,5)}$ demonstrating phenotypic differences. Metronidazole (MTZ) is the first-line prescribed drug with various rates of efficacy ranging from $0 \%$ to $100 \%$ depending on the dose administered ${ }^{(6)}$. Although MTZ demonstrates effectiveness in some individuals ${ }^{(7)}$, it has also been shown to exhibit side effects and resistance in others $^{(8)}$. MTZ induces apoptosis in Blastocystis spp. as a defensive mechanism to ensure that some of the cells survive to propagate the genome ${ }^{(9)}$.

Accumulating epidemiological data suggest that blastocytosis is associated with various gastrointestinal conditions including $\mathrm{CRC}^{(10,11)}$. Incidence of blastocytosis was $36 \%$ in healthy individuals and $34 \%$ in patients with colorectal adenoma, increasing significantly to $53 \%$ in patients with $\mathrm{CRC}^{(10)}$. The existence of extreme genotypic diversity among Blastocystis isolates from different clinical groups of carriers, requires the investigation of possible different biological and phenotypic characters between isolates.

Because of this diversity, the aim of the present work was to use growth kinetics (GK) assay and MTZ sensitivity as a platform to evaluate the phenotypic profile of Blastocystis isolates from CRC and non-CRC patients.

\section{MATERIAL AND METHODS}

This case-control study was conducted during the period from September, 2015 to October, 2017. The study was approved by the Ethics Board of Ain Shams University.

Blastocystis spp. isolates: A total of 19 Blastocystis spp. isolates from CRC and apparent non-CRC patients attending the outpatient clinics of El Demerdash 
Hospitals of Faculty of Medicine, Ain Shams University were studied. Blastocystis spp. isolates were sorted in 3 groups: 7 isolates from early diagnosed CRC patients before receiving any chemotherapeutic drugs (GI or CRC group), and 12 isolates from apparent non-CRC carriers (GII or non-CRC group). GII included 6 isolates from symptomatic patients with gastrointestinal tract symptoms suggestive of blastocystosis (GIIA), and 6 isolates from asymptomatic carriers (GIIB).

Blastocystis spp. were isolated by in-vitro cultivation of stool samples in Locke's Egg (LE) medium supplemented with $10 \%$ bovine serum and antibiotics at $37^{\circ} \mathrm{C}^{(12)}$. Subsequently, parasites were maintained in the laboratory by sub-culturing every 3 to 4 days when organisms were in the log phase of growth.

Growth characteristics: Starting with an initial concentration of $1 \times 10^{4}$ cells $/ \mathrm{ml}$ in LE medium, GK studies were performed in triplicate for each isolate. Viable Blastocystis organisms were counted daily in the Improved Neubauer hemocytometer chamber (Haussler Scientific) using Trypan blue viability exclusion test ${ }^{(13)}$ where $0.4 \%$ Trypan blue solution was used as an indicator of viability. Only viable organisms that exclude the dye and appear with clear cytoplasm were counted. Counting continued till no viable organisms were detected in culture tubes. Average counts of isolates in each group were calculated at 24 $\mathrm{h}$ intervals and an average growth kinetic curve was drawn for each group. The generation time (GT) was calculated in the first $24 \mathrm{~h}$ period during which the most rapid growth occurred, according to the following formula $^{(14)}: \mathrm{GT}=\mathrm{t} / 3.3 \log (\mathrm{b} / \mathrm{B})$, where: $\mathrm{t}=$ time period, $\log =$ logarithm to base 10 (common log), b: number of Blastocystis organisms at the end of time period $t$, and $\mathrm{B}=$ number of Blastocystis organisms at start of time period $t$.

MTZ sensitivity assay ${ }^{(15)}$ : Aqueous working solution of $1 \mathrm{mg} / \mathrm{ml} \mathrm{MTZ}$ was prepared and added to the medium to give final concentrations of 10, 25, 50, 100 and 200 $\mu \mathrm{g} / \mathrm{ml}$ medium. A duplicate of each concentration was done. An initial Blastocystis organisms' concentration of $5.0 \times 10^{5} \mathrm{org} / \mathrm{ml}$ medium was incubated at $37^{\circ} \mathrm{C}$ with the different MTZ concentrations. The percentage increase or decrease in growth in the test tubes and the control tubes (with no drug added) were calculated every $24 \mathrm{~h}$. The minimum inhibitory concentrate (MIC) and the minimal lethal concentrate (MLC) were identified for each isolate.

Statistical analysis: Results are expressed as mean \pm standard deviation (SD) for quantitative values and as number and percent for qualitative values. Statistical analyses were carried out using SPSS version 20. Oneway test of ANOVA was used for comparison of growth characteristic and MTZ sensitivity of the isolates. Significance level was considered at probability $P<0.05$.

\section{RESULTS}

Growth characteristics: Blastocystis spp. vacuolar form was detected in culture of all 19 isolates $(100 \%)$ while the amoeboid form was detected in $3 / 7(43 \%)$ of isolates of CRC group (GI), and 2/6 (33\%) of isolates of non-CRC symptomatic group (GIIB); and was not detected at all in cultures of non-CRC asymptomatic carriers (GIIA) with no statistical significant difference between the groups (Table 1).

Three distinct and different growth profiles representing average growth kinetics of isolates in the three groups starting with an initial inoculation of $1.0 \times 10^{4}$ cells $/ \mathrm{ml}$ are shown in figure (1). No viable organisms were found after $168 \mathrm{~h}$ (7 days) incubation in all isolates in all groups (Figure 1). CRC isolates in GI and symptomatic isolates in GIIA showed nearly similar average higher growth peaks compared to the asymptomatic isolates GIIB (Figure 2). Peak counts in the three groups were reached after $72 \mathrm{~h}$ incubation (Figure 1), with the lowest values achieved for the asymptomatic isolates in GIIB reaching 188 to $235 \mathrm{x}$ $10^{4}$ organisms/ml (Figure 2). Higher peak counts were those of CRC isolates in GI isolates, ranging from 213 to $302 \times 10^{4}$ organisms $/ \mathrm{ml}$, and of non-CRC symptomatic isolates in GIIA with values ranging from 217 to $301 \mathrm{x}$ $10^{4}$ organisms/ml (Figure 2 ).

The generation time recorded in the first $24 \mathrm{~h}$ for all isolates showed that CRC isolates (GI) $(8.97 \pm 1.16 \mathrm{~h})$ and isolates (GIIA) $(8.89 \pm 1.21 \mathrm{~h})$ were nearly similar

Table 1. Frequency of Blastocystis spp. forms in culture tubes of isolates from different groups studied.

\begin{tabular}{|c|c|c|c|c|c|}
\hline \multirow{5}{*}{$\begin{array}{c}\text { Blastocystis } \\
\text { forms in culture }\end{array}$} & \multicolumn{4}{|c|}{ Studied groups } & \multirow{4}{*}{$\begin{array}{l}\text { Statistical } \\
\text { analysis }\end{array}$} \\
\hline & \multirow{2}{*}{ CRC } & \multicolumn{2}{|c|}{ Non-CRC (GII) } & \multirow{3}{*}{ Total } & \\
\hline & & Symptomatic & Asymptomatic & & \\
\hline & GI & GIIA & GIIB & & \\
\hline & No. (\%) & No. (\%) & No. (\%) & No. (\%) & $P$ value \\
\hline Vacuolar & 7 (100) & $6(100)$ & $6(100)$ & $19(100)$ & N A \\
\hline Granular & $4(57)$ & $5(83.3)$ & $3(50)$ & $12(63)$ & 0.448 \\
\hline Amoeboid & $3(43)$ & $2(33.3)$ & $0(0)$ & $5(26.3)$ & 0.194 \\
\hline
\end{tabular}

CRC: Colorectal carcinoma; NA: Not available as it is constant. 
to non-CRC symptomatic (Table 2). Both were faster in growth than asymptomatic isolates (GIIB) $(9.55 \pm 0.31$ h). Both GI and GIIA achieved parasite counts greater than in asymptomatic group at peak growth.

MTZ susceptibility assay (Tables 3,4 , and 5 and Figures 3 and 4): The number of organisms in the control tubes with no drug added and incubated under the same culture conditions, started to decline after $48 \mathrm{~h}$ incubation (data not shown). For that reason, the assay was carried out over 2 days duration only. Great reduction (GI, 94.21\%, GIIA, 90.31\% and GIIB, 94.13\%) in growth of all Blastocystis isolates in the three groups occurred during the first $24 \mathrm{~h}$ exposure to different doses of MTZ varying between $10 \mu \mathrm{g} / \mathrm{ml}$ to $200 \mu \mathrm{g} / \mathrm{ml}$. This progressed for every isolate till the end of $48 \mathrm{~h}$ of the assay. GIIA (non-CRC symptomatic isolates) were the least affected than the isolates in GI (CRC group) and in GIIB (non-CRC asymptomatic sub-group). MIC value for all the isolates was $200 \mu \mathrm{g} / \mathrm{ml}$. No minimal lethal dose (MLC) was recorded as no complete death of organisms occurred in culture.

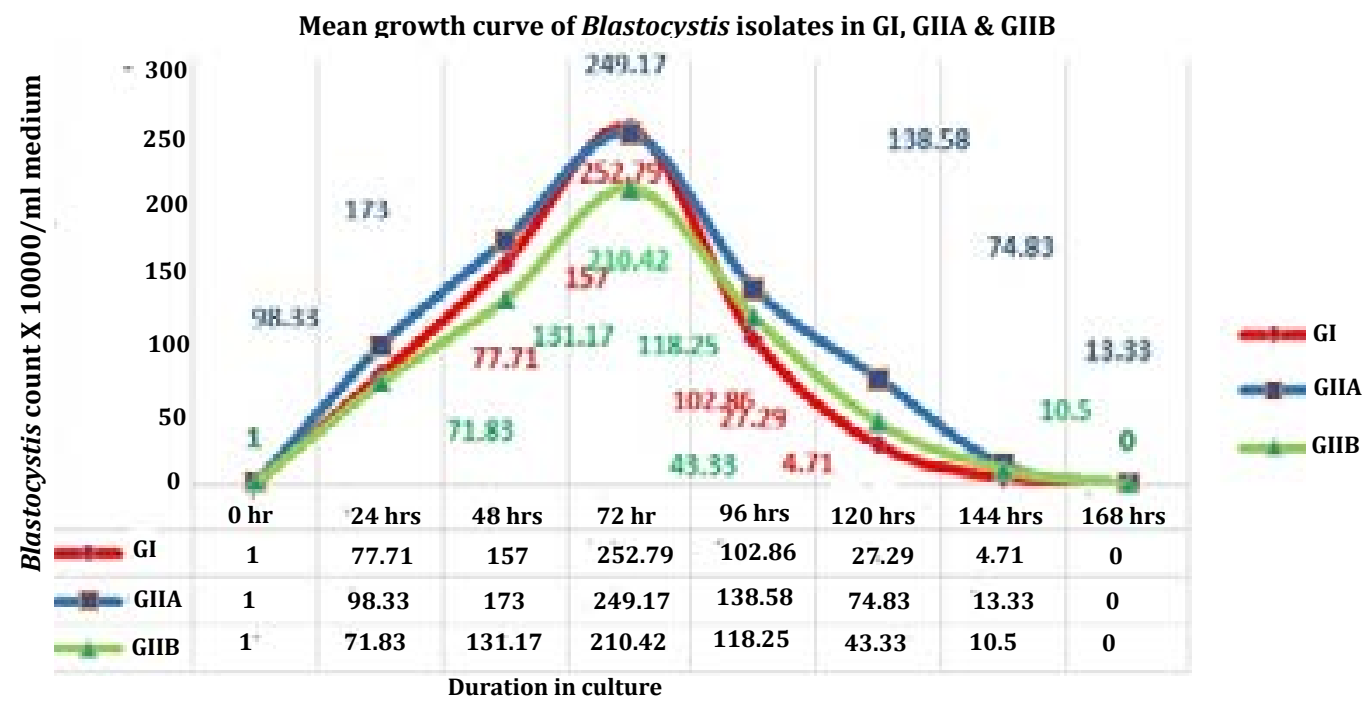

Fig. 1. Line chart for the growth kinetics of Blastocystis isolates of GI, GIIA and GIIB organisms in the biphasic LE culture medium at $37^{\circ} \mathrm{C}$ incubation starting by Blastocystis organisms' concentration of $1.0 \times 10^{4} / \mathrm{ml}$.

GI: CRC group, GIIA: non-CRC symptomatic subgroup, GIIB: non-CRC asymptomatic subgroup.

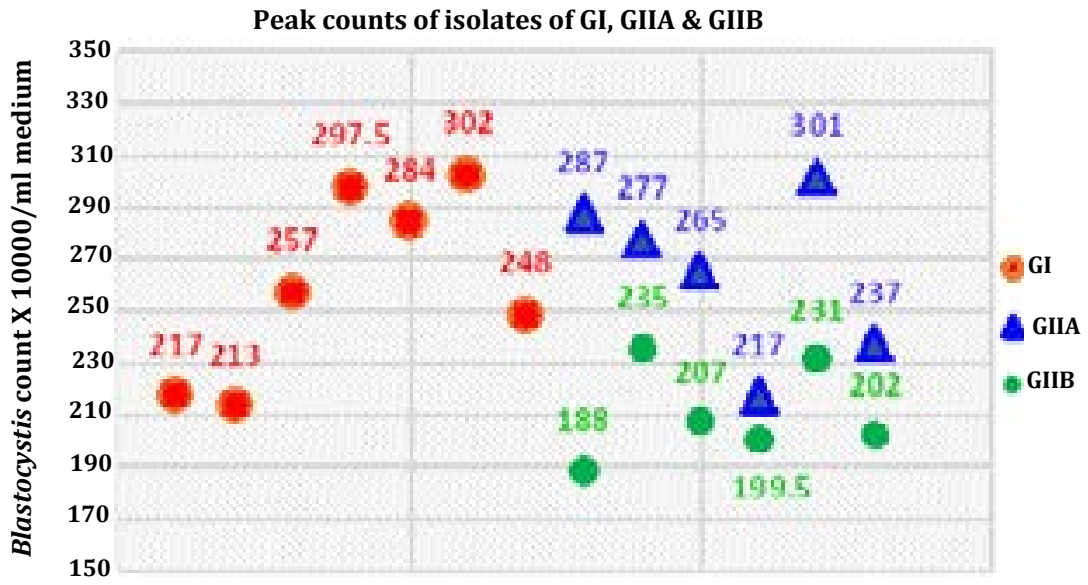

Fig. 2. Scatter chart for the growth peaks of isolates of the three groups.

GI: CRC group, GIIA: non-CRC symptomatic subgroup, GIIB: non-CRC asymptomatic subgroup.

Table 2. Mean generation time of Blastocystis isolates in GI, GIIA and GIIB.

\begin{tabular}{|c|c|c|c|c|c|}
\hline & CRC (GI) & Non-CRC symptomatic (GIIA) & Non-CRC asymptomatic (GIIB) & \multicolumn{2}{|c|}{ Statistical analysis } \\
\hline & Mean \pm SD & Mean \pm SD & Mean \pm SD & $\mathbf{F}$ & $P$ value \\
\hline \multicolumn{6}{|c|}{ Generation time (Hour) } \\
\hline Mean \pm SD & $8.97 \pm 1.16$ & $8.89 \pm 1.21$ & $9.55 \pm 0.31$ & \multirow{2}{*}{0.804} & \multirow{2}{*}{0.465} \\
\hline Range & $6.4-9.63$ & $6.43-9.65$ & $9.2-10.0$ & & \\
\hline
\end{tabular}

Comparison using One-way ANOVA test 
Table 3. Mean counts of viable Blastocystis organisms/ml in culture of isolates of GI (CRC group), GIIA (non-CRC symptomatic subgroup) and GIIB (non-CRC asymptomatic subgroup) after 24 and $48 \mathrm{~h}$ exposure to $200 \mu \mathrm{g} / \mathrm{ml}$ concentration of MTZ (starting counts $5.0 \times 10^{5}$ organisms $\left./ \mathrm{ml}\right)$.

\begin{tabular}{|c|c|c|c|c|c|}
\hline & \multirow{2}{*}{ CRC (GI) } & \multirow{2}{*}{ Non-CRC symptomatic (GIIA) } & \multirow{2}{*}{ Non-CRC asymptomatic (GIIB) } & \multicolumn{2}{|c|}{ Statistical analysis } \\
\hline & & & & $\mathbf{F}$ & $P$ value \\
\hline Mean \pm SD & $6.36 \pm 2.23$ & $10.08 \pm 2.58^{*}$ & $7.83 \pm 2.64$ & \multirow{2}{*}{2.672} & \multirow{2}{*}{$0.023^{*}$} \\
\hline Range (after $24 \mathrm{~h}$ ) & $3-9$ & $8-15$ & $4-12$ & & \\
\hline Mean \pm SD & $4.64 \pm 1.75$ & $6.75 \pm 2.09 *$ & $5.17 \pm 2.32$ & \multirow{2}{*}{2.196} & \multirow{2}{*}{$0.038^{*}$} \\
\hline Range (after $48 \mathrm{~h}$ ) & $2-7$ & $4-10$ & $3-9$ & & \\
\hline
\end{tabular}

Comparison by the One-way ANOVA test, ${ }^{*}=$ significant difference.

Table 4. Percentage decrease in mean counts of isolates of GI (CRC group), GIIA (non-CRC symptomatic sub-group) and GIIB (nonCRC asymptomatic sub-group) in culture after $24 \mathrm{~h}$ exposure to different concentrations of MTZ.

\begin{tabular}{|c|c|c|c|c|c|}
\hline \multirow{2}{*}{$\begin{array}{c}\text { Drug } \\
\text { Concentrations }\end{array}$} & \multicolumn{3}{|c|}{ Percentage decrease of counts after $24 \mathrm{~h}$} & \multicolumn{2}{|c|}{ Statistical analysis } \\
\hline & CRC (GI) & Non-CRC symptomatic (GIIA) & Non-CRC asymptomatic (GIIB) & $\mathbf{F}$ & $P$ value \\
\hline $10 \mu \mathrm{g} / \mathrm{ml}$ & $82.97 \%$ & $83.65 \%$ & $86.81 \%$ & 1.921 & 0.179 \\
\hline $25 \mu \mathrm{g} / \mathrm{ml}$ & $85.04 \%$ & $85.17 \%$ & $89.25 \%$ & 0.997 & 0.391 \\
\hline $50 \mu \mathrm{g} / \mathrm{ml}$ & $88.10 \%$ & $87.74 \%$ & $91.19 \%$ & 0.490 & 0.622 \\
\hline $100 \mu \mathrm{g} / \mathrm{ml}$ & $91.16 \%$ & $89.11 \%$ & $92.75 \%$ & 1.925 & 0.178 \\
\hline $200 \mu \mathrm{g} / \mathrm{ml}$ & $94.21 \%$ & $* 90.31 \%$ & $94.13 \%$ & 2.196 & $0.038^{*}$ \\
\hline
\end{tabular}

* Significant difference.

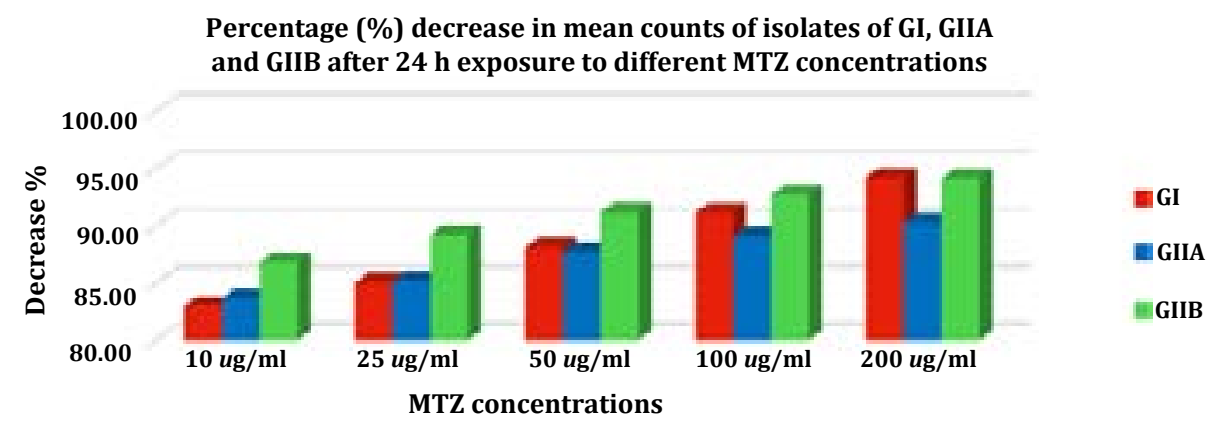

Fig. 3. Bar chart for the percentage decrease in mean counts of isolates of GI (CRC group), GIIA (non-CRC symptomatic subgroup) and GIIB (non-CRC asymptomatic subgroup) in culture after $24 \mathrm{~h}$ exposure to different concentrations of MTZ.

Table 5. Percentage (\%) decrease in mean counts of isolates of GI (CRC group), GIIA (non-CRC symptomatic sub-group) and GIIB (non-CRC asymptomatic sub-group) in culture after $48 \mathrm{~h}$ exposure to different concentrations of MTZ.

\begin{tabular}{|c|c|c|c|c|c|}
\hline \multirow{2}{*}{$\begin{array}{c}\text { Drug } \\
\text { Concentrations }\end{array}$} & \multicolumn{3}{|c|}{ Percentage decrease of counts after $48 \mathrm{~h}$} & \multicolumn{2}{|c|}{ Statistical analysis } \\
\hline & CRC (GI) & Non-CRC symptomatic (GIIA) & Non-CRC asymptomatic (GIIB) & $\mathbf{F}$ & $P$ value \\
\hline $10 \mu \mathrm{g} / \mathrm{ml}$ & 91.75 & 91.52 & 93.07 & 1.921 & 0.087 \\
\hline $25 \mu \mathrm{g} / \mathrm{ml}$ & 91.75 & 91.52 & 93.07 & 0.997 & 0.179 \\
\hline $50 \mu \mathrm{g} / \mathrm{ml}$ & 94.94 & 93.78 & 95.62 & 0.490 & 0.391 \\
\hline $100 \mu \mathrm{g} / \mathrm{ml}$ & 96.50 & $94.41^{*}$ & 96.36 & 1.925 & 0.178 \\
\hline $200 \mu \mathrm{g} / \mathrm{ml}$ & 97.84 & $96.35^{*}$ & 97.77 & 2.196 & $0.038^{*}$ \\
\hline
\end{tabular}

* Significant difference.

Fig. 4. Bar chart for percentage decreases in the mean organism counts of isolates of GI (CRC group), GIIA (non-CRC symptomatic subgroup) and GIIB (non-CRC asymptomatic subgroup) after $48 \mathrm{~h}$ exposure to different concentration of MTZ.
Percentage (\%) decrease in mean counts of isolates of GI, GIIA and GIIB after $48 \mathrm{~h}$ exposure to different MTZ concentrations

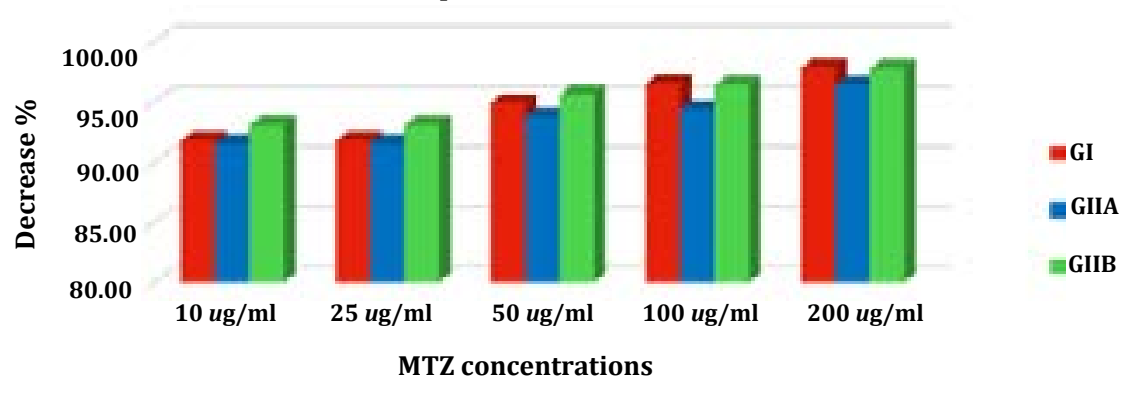




\section{DISCUSSION}

In the present study, the presence of amoeboid forms in culture of $43 \%$ of CRC isolates (GI) and $33.3 \%$ of nonCRC symptomatic isolates (GIIA) and its complete absence in asymptomatic isolates (GIIB) may be indicative of virulence in some of the isolates in GI and GIIA. This is consistent with previous studies ${ }^{(4,16,17)}$ in which significant presence of amoeboid forms was recorded in cultures from symptomatic cases only and not in cultures from asymptomatic groups. However, Souppart et al. ${ }^{(18)}$ detected the amoeboid form in culture of both symptomatic and asymptomatic Blastocystis infected groups with no significant difference.

Three different growth profiles were detected in the studied groups. Similarly, different growth profiles of isolates from different clinical groups were previously compared $^{(4,5)}$ revealing differences between isolates recruited from different clinical groups.

In the present study, higher peak growth values of symptomatic isolates of GIIA than the asymptomatic isolates of GIIB differ with the findings of some previous studies ${ }^{(4,5)}$, in which a higher growth of asymptomatic over symptomatic isolates was reported reaching peak values after $5 \mathrm{~d}$ incubation. This may be attributed to combined factors related to variation in constituents of the Jonnes' culture medium used, and the different biological behaviors of the isolates. Also, it should be considered that the associating microbiota in gut of Egyptians on the growth of asymptomatic isolates in the present study could play a role in inhibiting growth of Blastocystis in vitro.

In the report by Ragavan et $a l^{(5)}$, a third group of isolates derived from patients with irritable bowel syndrome (IBS) were included in the study, and this group had intermediate peak values between the symptomatic and asymptomatic groups. In our present study, the third group of isolates consisting of CRC isolates presented a nearly similar curve to that of the subgroup of symptomatic isolates. It is noteworthy to mention that average peak counts and growth curves of isolates from groups GI and GIIA were approximately similar and both were higher than isolates from group GIIB.

The generation time recorded in the present study indicated that CRC isolates of GI achieved nearly similar timing as non-CRC symptomatic GIIA isolates, both being faster in growth than asymptomatic GIIB isolates, and thus, both GI and GIIA achieved parasite counts greater than asymptomatic isolates at peak growth. Other studies ${ }^{(14-20)}$ reported that the generation time of xenic Blastocystis isolates was variable, ranging from 6 to $23 \mathrm{~h}$, depending on the isolate itself and the type of medium used.

In the present work, in vitro MTZ sensitivity assay was performed and showed that great reduction occurred in the three groups within $24 \mathrm{~h}$, and progressed till the end of $48 \mathrm{~h}$ of the assay. Non-CRC symptomatic GIIA isolates were the least affected as compared to CRC isolates GI group and in asymptomatic sub-group GIIB. Thus, the symptomatic virulent isolates of GIIA showed less MTZ sensitivite $\left(\mathrm{MTZ}^{\mathrm{s}}\right)$. This contrasts with what was expected to occur where as reported virulent Blastocystis isolates are more $\mathrm{MTZ}^{\mathrm{s}}$ than avirulent isolates ${ }^{(8,21)}$.

In the present study, isolates in the CRC group (GI), were nearly as sensitive as the non-CRC asymptomatic isolates of GIIB, and both were more $\mathrm{MTZ}^{\text {s }}$ than the nonCRC symptomatic isolates of GIIA. This may be explained by the biological variability of isolates providing a possible explanation for the diverse clinical outcomes of blastocystosis. On comparing the GK of the isolates in the three groups studied GI, GIIA and GIIB and the results of MTZ susceptibility assay, the GIIA and GIIB isolates were both less MTZ; indicating that the more MTZ-resistant $\left(\mathrm{MTZ}^{\mathrm{r}}\right)$, the more rapid the growth. This observation was approached by $\mathrm{Wu}$ et al., ${ }^{(21)}$ who examined the proliferative potential of $\mathrm{MTZ}^{\mathrm{r}}$ and $\mathrm{MTZ}^{\mathrm{s}}$ strains from growth curves assays. They noticed that the growth rates of MTZ ${ }^{\mathrm{r}}$ isolates were not always lower than $\mathrm{MTZ}^{\mathrm{s}}$ isolates, suggesting that drug resistance in Blastocystis might not necessarily be associated with slower growth. The isolates of GIIB asymptomatic isolates, are apparently avirulent, producing no-symptoms in their hosts, and are $\mathrm{MTZ}^{\mathrm{s}}$, and slow growing. While GIIA symptomatic isolates were rapidly growing and less $\mathrm{MTZ}^{\mathrm{s}}$, and the CRC isolates were rapidly growing and $\mathrm{MTZ}^{\text {s. }}$.

In the current study, MTZ caused only inhibition of in vitro growth of all the isolates, an observation that is in accordance with the description of Zierdt et al., ${ }^{(22)}$ who reported that MTZ is one of the inhibitory antimicrobials and not a lethal drug. This, also, agrees with the reports of Nasirudeen et al., ${ }^{(23)}$ who explained that MTZ induces apoptosis in Blastocystis spp. as a defensive mechanism used by unicellular organisms for the preservation of cell populations to ensure that some of the cells survive to propagate the genome ${ }^{(9)}$. The survival of resistant isolates could be a result of an efficient and effective apoptotic response to MTZ with the formation of granular forms and later release of new progeny of vacuolar forms contained in their vacuoles ${ }^{(23)}$. Also, resistance of strains may be due to insufficient dosage of $\mathrm{MTZ}^{(24)}$. Thus, Anselmi et al., ${ }^{(24)}$ reported that further research is needed to investigate dose-dependent effects and the possibility of a lethal effect if higher concentrations of the drug are used..

In conclusion, studying the growth kinetics and MTZ sensitivity in the present study demonstrated the existence of differences in the biological characters of Blastocystis spp. isolates from different clinical groups a possible indication of different pathogenic roles.

Author contribution: FSM Habib designed the study, analyzed the data, and wrote the manuscript. NS AbdelFattah shared in designing the study and conceived the culture, and analyzed the data. GA Saad shared in 
performing the culture, and analysis of data. HM El Naggar collected the isolates, performed the culture.

Conflict of interest: There is no conflict of interest.

Financial support and sponsorship: Nil.

\section{REFERENCES}

1. Tan KS. Blastocystis in humans and animals: new insights using modern methodologies. Vet Parasitol 2004; 126:121-144.

2. Abaza SM, Rayan HZ, Soliman RH, Nemr NA, Mokhtar AB. Subtype analysis of Blastocystis spp. isolates from symptomatic and asymptomatic patients in Suez Canal University Hospitals, Ismailia, Egypt. PUJ 2014; 7(1):5667.

3. Stensvold CR, Lewis HC, Hammerum AM, Porsbo LJ, Nielsen SS, Olsen KE, et al. Blastocystis: unravelling potential risk factors and clinical significance of a common but neglected parasite. Epidemiol Infect 2009; 137: $1655-1663$.

4. Tan TC, Suresh KG, Smith HV. Phenotypic and genotypic characterization of Blastocystis hominis isolates implicates subtype 3 as a subtype with pathogenic potential. Parasitol Res 2008; 104:85-93.

5. Ragavan ND, Govind SK, Chye TT, Mahadeva S. Phenotypic variation in Blastocystis sp. ST3. Parasites \& Vectors 2014; 7: 404.

6. Kurt Ö. Eradication of Blastocystis in humans: Really necessary for all? Parasitol Intern 2016; 65(6): 797-801.

7. Moghaddam DD, Ghadirian E and Azami M. Blastocystis hominis and the evaluation of efficacy of metronidazole and trimethoprim/sulfamethoxazole. Parasitol Res 2005; 96: 273-275.

8. Mirza H, Wu Z, Kidwai F, Tan KS. A metronidazoleresistant isolate of Blastocystis spp. is susceptible to nitric oxide and down-regulates intestinal epithelial inducible nitric oxide synthase by a novel parasite survival mechanism. Infect. Immun 2011; 79:50195026.

9. Dhurga DB, Suresh KG, Tan TC, Chandramathi S. Apoptosis in Blastocystis spp. is 392 related to subtype. Trans R Soc Trop Med Hyg 2012; 106:725-730.

10. Steer H. Blastocystis hominis and colorectal cancer. Ann R Coll Surg Engl 2007; 89: 538-539.

11. Padukone S, Mandal J, Parija AC. Severe Blastocystis subtype 3 infection in a patient with colorectal cancer. Trop Parasitol 2017; 7(2):122-124.
12. Zierdt CH. Blastocystis hominis: Past and future. Clin Microbio Rev 1991; 4:61-79.

13. Fauque $\mathrm{P}$, Ben Amor A, Joanne C, Agnani G, Bresson JL, Roux C. Use of trypan blue staining to assess the quality of ovarian cryopreservation. Fertil Steril 2007;87:1200-1207.

14. Zierdt $\mathrm{CH}$, Swan JC. Generation time and growth rate of the human intestinal parasite Blastocystis hominis. J Protozool 1981; 28:483-485.

15. Yakoob J, Jafri W, Jafri N, Islam M and Beg MA. In vitro susceptibility of Blastocystis hominis isolated from patients with irritable bowel syndrome. Br J Biomed Sci 2004; 61:75-77.

16. Tan TC, Suresh KG. Predominance of amoeboid forms of Blastocystis hominis in isolates from symptomatic patients. Parasitol Res 2006; 98:189-193.

17. Vassalos CM, Spanakos G, Vassalou E, Papadopoulou C, Vakalis N. Differences in clinical significance and morphologic features of Blastocystis spp. subtype 3. Am J Clin Pathol 2010; 133:251-258.

18. Souppart L, Sanciu G, Cian A, Wawrzyniak I, Delbac F, Capron $\mathrm{M}$, et al. Molecular epidemiology of human Blastocystis isolates in France. Parasitol Res 2009; 105:413-421.

19. Boreham PFL, Stenzel DJ. Blastocystis in humans and animals: morphology, biology and epizootiology. Adv Parasitol 1993; 32:1-70.

20. Irikov OA, Antokhin AI, Romanov YA. Study of the dynamics of Blastocystis hominis reproduction in vitro. Bull Exp Biol Med 2009; 148:99-102.

21. Wu Z, Mirza $\mathrm{H}$, Tan KS. Intra-subtype variation in entero-adhesion accounts for differences in epithelial barrier disruption and is associated with metronidazole resistance in Blastocystis subtype-7. PLoS Negl Trop Dis 2014; 8: 2885.

22. Zierdt $\mathrm{C} \mathrm{H}$, Swan J $\mathrm{C}$ and Hosseini J. In vitro response of Blastocystis hominis to antiprotozoal drugs. J Protozool 1983; 30:332-334.

23. Nasirudeen AM, Hian YE, Singh M, Tan KS. Metronidazole induces programmed cell death in the protozoan parasite Blastocystis hominis. Microbiology 2004; 150:33-43.

24. Anselmi C, Ettorre A, Andreassi M, Centini M, Neri P, Di Stefano A. In vitro induction of apoptosis vs. necrosis by widely used preservatives: 2-phenoxyethanol, a mixture of Iso-thiazolinones, imidazolidinyl urea and 1, 2-pentanediol. Biochem. Pharmacol 2002; 63: 437453. 